\title{
Avaliação de Habilidades e Competências do Século XXI a partir do Comportamento do Estudante em Jogos Digitais
}

\author{
Handerson B. Medeiros ${ }^{1}$, Isabel D. Nunes ${ }^{2}$, Eduardo Aranha ${ }^{1}$ \\ ${ }^{1}$ Departamento de Informática e Matemática Aplicada - Universidade Federal do Rio \\ Grande do Norte (UFRN) \\ ${ }^{2}$ Instituto Metrópole Digital - Universidade Federal do Rio Grande do Norte (UFRN) \\ handersonmedeiros@gmail.com; bel@imd.ufrn.br; \\ eduardoaranhaddimap.ufrn.br
}

\begin{abstract}
The new guidelines of the National Curriculum Parameters show the need for an education based on skills and abilities. Cognitive skills such as classifying and applying rules should be considered, but also social-emotional skills such as curiosity and perseverance are needed. This article discusses the challenge of using digital games in the teaching and learning process and how to assess such skills. Techniques such as ECD (Evidence-Centred Design), Artificial Intelligence and Data Mining can be used for this purpose, as long as their specificities are considered and the difficulties in obtaining data from the use of a digital game are overcome.
\end{abstract}

Resumo. As novas diretrizes dos Parâmetros Curriculares Nacionais mostram a necessidade de um ensino baseado em competências e habilidades. Habilidades cognitivas, tais como classificar e aplicar regras devem ser consideradas, mas também são necessárias habilidades socioemocionais, como curiosidade e perseverança. Este artigo discute o desafio do uso de jogos digitais no processo de ensino e aprendizagem e como avaliar tais habilidades. Técnicas como a ECD (Evidence-Centred Design), inteligência artificial e mineração de dados podem ser utilizadas para esse fim, desde que sejam consideradas suas especificidades e superadas as dificuldades em obtenção de dados a partir do uso de um jogo digital.

\section{Introdução}

Segundo Gatti (2005), a principal preocupação das escolas, pais e, sobretudo a dos alunos, reside em descobrir quais as competências que os jovens precisam aprender hoje para alcançarem sucesso acadêmico, profissional e pessoal. As atuais diretrizes para educação, tal como Parâmetros Curriculares Nacionais ${ }^{1}$, apontam para uma nova forma de ensino/aprendizagem, exigindo a mudança da tradicional transmissão do conhecimento e de memorização para um cenário com base nas competências e habilidades a serem desenvolvidas [Cotta et al. 2015]. Dados da Organização para Cooperação e Desenvolvimento Econômico (OCDE) encontraram que não somente as habilidades cognitivas (identificar e classificar conceitos, aplicar regras, resolver

\footnotetext{
${ }^{1}$ https://goo.gl/7fbGe7
} 
problemas e etc), mas também as não cognitivas ou socioemocionais tais como sociabilidade, curiosidade, perseverança, influenciavam fortemente as decisões educacionais [OCDE 2015] são importantes na construção dos saberes.

Uma forma de trazer e integrar o desenvolvimento de tais habilidades é a introdução de atividades lúdicas como jogos digitais, fornecendo uma metodologia de ensino motivadora, possibilitando uma prática significativa daquilo que está sendo aprendendo e potencializando a construção e avaliação do conhecimento. Gatti (2005) afirma que o indivíduo se constitui e se desenvolve na medida em que interage e é estimulado pelo meio. Os jogos estimulam a aprendizagem, pois permitem a interação e construção coletiva de conceitos e experiências, bem como a geração de conhecimento [Prensky 2012].

Avaliar a aprendizagem significa determinar as competências adquiridas por meio de aspectos quantitativos e qualitativos. Essa avaliação é um processo que envolve a coleta, medição e interpretação de dados. Com os valores coletados é realizada uma medição dos dados baseada em um conjunto de critérios, qualificando os resultados daqueles que foram avaliados. Interpretando tais resultados pode-se quantificar o grau de qualidade ou características que o discente possui, isso pode refletir diretamente na tomada de decisão dos educadores e determinar cada passo do processo ensino-aprendizagem.

Neste sentido, este artigo apresenta direcionamentos para pesquisas em avaliações de habilidades do século XXI no contexto dos jogos digitais a fim de incentivar a busca de soluções para os desafios existentes.

\section{Habilidades e competências do século XXI}

O conhecimento aprendido deve ser relembrado de forma constante para que seja mantido na memória, podendo assim, ser utilizado em atividades de interpretação e de raciocínio lógico [Rosa e Galvão 2015]. Cotta et al. (2015) também enfatiza que os alunos devem saber usar a informação e o conhecimento adquirido na aplicação de situações novas e complexas, de forma autônoma, sabendo "Fazer", "Conviver" e "Ser", como mostra a Figura 1.

Não basta dominar Português e Matemática se o indivíduo não souber se relacionar com os outros, não for determinado e não conseguir controlar suas emoções, entre outras características da personalidade. As competências socioemocionais, também chamadas competências não cognitivas, são habilidades utilizadas na realização de tarefas que envolvam trabalho em grupo e controle emocional, como: autonomia, estabilidade emocional, sociabilidade, capacidade de superar fracassos, curiosidade, perseverança. O Instituto Ayrton Senna e a Organização para Cooperação e Desenvolvimento Econômico (OCDE) fizeram um estudo com uma ferramenta desenvolvida para a medição de competências socioemocionais, agrupando as habilidades cognitivas e não cognitivas mais importantes para serem avaliadas, como mostra a Figura 2. 


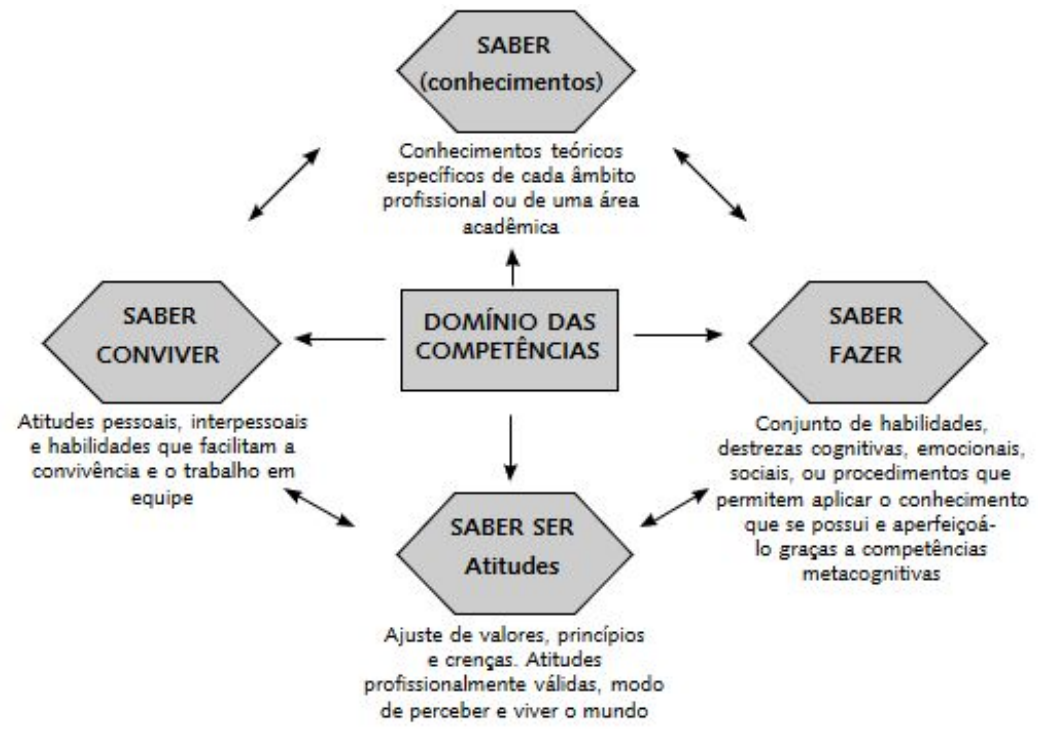

Figura 1. Domínio das competências a um aprendizado constante. Fonte: [Cotta et al. 2015]

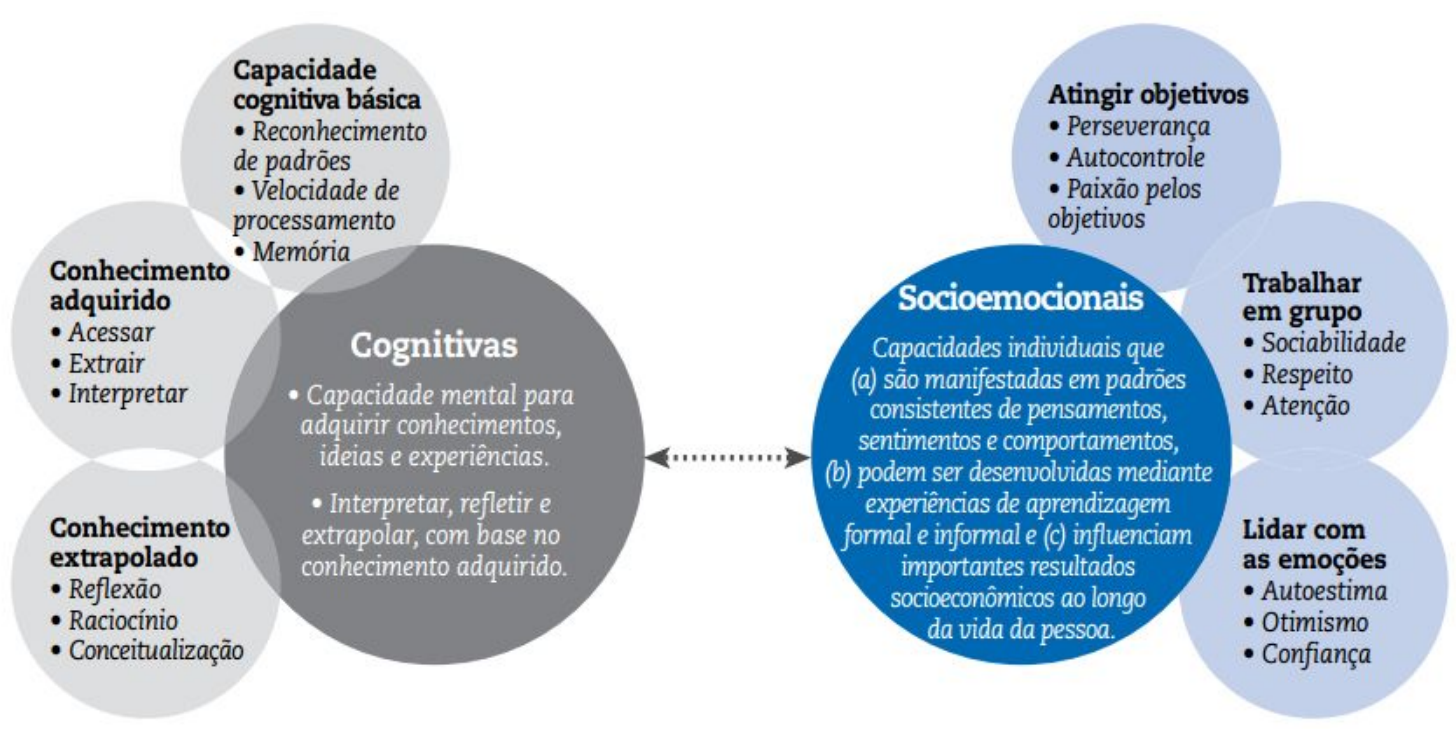

Figura 2. Descrição das habilidades cognitivas e socioemocionais [OCDE 2015].

\subsubsection{Habilidades Curriculares}

$\mathrm{Na}$ educação formal é exigida do professor a verificação e mensuração do aprendizado do aluno, apresentando quantitativamente os resultados da aprendizagem. E esses, por sua vez, são obtidos aplicando provas e testes regulares nas instituições do governo e programas e ações do Ministério da Educação brasileiro².

O governo federal utiliza o Indicador de Desenvolvimento da Educação Básica (IDEB) para medir a qualidade do ensino nas escolas públicas. Seu cálculo é realizado a

\footnotetext{
${ }^{2}$ Ministério da Educação, avaliações da aprendizagem: https://goo.gl/JtVGcU
} 
partir dos dados sobre aprovação escolar, obtidos no Censo Escolar, e das médias de desempenho nas avaliações do Inep (Instituto Nacional de Estudos e Pesquisas Educacionais Anísio Teixeira), o Sistema de Avaliação do Ensino Básico (SAEB), composto por: a Avaliação Nacional da Educação Básica (Aneb), a Avaliação Nacional do Rendimento Escolar (Anresc), conhecida como Prova Brasil, e a Avaliação Nacional da Alfabetização (ANA) e o Exame Nacional do Ensino Médio (ENEM).

Tais programas de avaliação implementados pelo governo brasileiro têm chamado a atenção para uma dimensão humana com uma longa tradição de pesquisa na Psicologia: as habilidades cognitivas. Comissões compostas para elaboração dessas provas, definem um conjunto de habilidades consideradas essenciais e, a partir delas, constroem os instrumentos de avaliação (INEP, 1999, p. 9). As avaliações estruturam-se a partir de matrizes de competências e habilidades que fundamentam a construção dos itens e informam como os autores entendem a inteligência e quais, entre as suas dimensões, devem ser privilegiadas pelo Ensino Básico e Médio.

De acordo com Council et al. (2013), estudos empíricos mostram que as competências cognitivas são capazes de explicar apenas uma pequena fração da associação entre educação e sucesso no trabalho e em outros aspectos da vida. Estudos conduzidos ao longo de quatro décadas afirmaram que a maior parte do impacto de anos de instrução sobre sucesso foi atribuída a habilidades socioemocionais e não apenas as habilidades cognitivas.

\subsubsection{Habilidades do Século XXI e fatores de habilidade e personalidade}

Um comitê formado por educadores, psicólogos e economistas pesquisou sobre o que se espera que os estudantes alcancem nos seus ciclos escolares, nos seus futuros trabalhos e em outros aspectos da vida [Council et al. 2013]. O resultado foi que as "habilidades do século XXI" são habilidades advindas dos desafios impostos ao estilo das novas tecnologias de informação e comunicação (TIC). As tecnologias estão cada vez mais presentes em sala de aula; o professor tem que se preocupar em preparar o aluno para ser atuante em um mundo em transformação e ajudá-lo a desenvolver as competências específicas para o século 21, definindo o que pode oferecer para que crianças e jovens tenham acesso à melhor formação possível [Perrenoud and Thurler 2009].

Segundo Council et al. (2013), as habilidades do século XXI foram divididas em três grandes domínios. O primeiro deles é o cognitivo, sendo aquele que envolve estratégias e processos de aprendizado, criatividade, memória, pensamento crítico; é o que está relacionado à aprendizagem mais tradicional. $\mathrm{O}$ intrapessoal tem relação com a capacidade de lidar com emoções e moldar comportamentos para atingir objetivos. Já o interpessoal envolve a habilidade de expressar ideias, interpretar e responder aos estímulos de outras pessoas.

As competências cognitivas estão agrupadas em três: I - Processos cognitivos e estratégias, tendo como principais termos: pensamento crítico, resolução de problema, análise, raciocínio / argumentação, interpretação, tomada de decisão, aprendizagem adaptativa; II - Conhecimento, tendo como principais termos: alfabetização em informação (pesquisa usando evidências e reconhecendo fontes confiáveis), alfabetização em tecnologia da informação e das comunicações, comunicação oral e escrita; escuta ativa; III - Criatividade e inovação. 
As competências intrapessoais estão divididas em mais três grupos: I Aperfeiçoamento intelectual, tendo como principais termos: iniciativa, autodirecionamento, responsabilidade, perseverança, produtividade, autorregulação tipo 1 (habilidades metacognitivas, incluindo previsão, desempenho e autorreflexão), profissionalismo, ética, integridade, orientação profissional; II - Auto avaliação do Núcleo Positivo: Auto-regulação tipo 2 (automonitoramento, auto avaliação, auto reforço), saúde física e psicológica.

Por fim, as competências interpessoais estão particionadas em dois grupos: I Habilidades sociais como trabalho em equipe e colaboração: comunicação, colaboração, trabalho em equipe, cooperação, coordenação, empatia, confiança, orientação de serviço, resolução de conflitos, negociação; e II - Habilidades sociais persuasivas como: Liderança, responsabilidade, comunicação assertiva, auto-representação, influência social com os outros.

\section{Revisão da literatura}

De acordo com Shute e Ventura (2013), existem várias razões para usar jogos na educação: I - as escolas permaneceram praticamente inalteradas por muitas décadas, enquanto o nosso mundo está mudando rapidamente, estamos vendo um número crescente de alunos desengajados; II - há uma necessidade de medidas dinâmicas e contínuas de processos de aprendizagem e resultados; III - muitos dos jogos normalmente exigem que um jogador aplique várias competências (por exemplo, criatividade, resolução de problemas, persistência e colaboração) para ter sucesso no jogo.

O trabalho de Serrano-Laguna (2017) realizou um apanhado de técnicas e padrões para sistematizar a análise de aprendizagem em jogos. Em geral, os jogos usam duas estratégias de interação: I - baseada em eventos, onde o jogo registra eventos pré-especificados quando ocorrem; e II - baseado no estado, onde o jogo repetidamente envia o estado do jogo a uma frequência específica. Serrano-Laguna (2017) destaca que em muitos casos, quando um jogador faz uma escolha em um jogo, ele precisaria aplicar conhecimento específico para tomar a decisão correta. As métricas comuns obtidas a partir dessas interações são: o tempo gasto para tomar decisões e a razão de correção/erro quando as seleções podem ser pontuadas positivamente ou negativamente (perguntas com uma resposta correta).

Schrier (2016) desenvolveu um jogo de Realidade Aumentada (AR) para ensinar habilidades do século XXI, como interpretação, resolução de problemas, gerenciamento de informações, trabalho em equipe, flexibilidade, engajamento cívico e aceitação de diversas perspectivas. Os participantes interagem com figuras e itens históricos virtuais ativados dependendo da localização do GPS no smartphone. Os testes iniciais sugerem que os jogos de AR, quando adequadamente projetados para fins pedagógicos, podem motivar a prática das habilidades do século XXI.

O Alien Rescue [Kang et al. 2017] utiliza jogos de mundo aberto juntamente com a pedagogia de aprendizagem baseada em problemas para resolver problemas. No trabalho são definidos dados de jogabilidade usados para identificar os padrões de comportamento dos alunos à medida que interagem com as ferramentas disponíveis no jogo. Este estudo examinou os frequentes padrões sequenciais entre estudantes de baixa 
e alta performance e mostrou que as estratégias de resolução de problemas eram diferentes entre esses dois grupos de atuação.

Zoo U [DeRosier e Thomas 2017] é um mundo escolar virtual que tem por objetivo apresentar situações sociais virtuais que as crianças experimentam na escola primária aproveitando a propensão natural das crianças em relação aos jogos para envolvê-los na resolução de problemas sociais e no pensamento crítico para a avaliação baseada no desempenho das habilidades. $\mathrm{O}$ presente estudo promove avaliação de habilidades socioemocionais (SE), examinando o grau da competência social diferenciando os alunos que experimentam problemas sociais de ajuste escolar. Utilizou-se uma abordagem implementada por designers de jogos educacionais conhecidos como Evidence Centered Design (ECD) [Almond et al. 2015] para avaliação de habilidades.

O trabalho [Shute et al. 2016] utilizou um jogo chamado Use Your Brainz (versão modificada de Plants vs. Zombies 2), para medir as habilidades de resolução de problemas dos estudantes do ensino médio. No jogo, os jogadores devem plantar uma variedade de plantas especiais em seu gramado para evitar que os zumbis entrem em sua casa. Criou-se uma rede Bayes exclusiva para cada nível de jogo, as estimativas relacionadas com a habilidade de resolução de problemas de um jogador são atualizadas à medida que as evidências em curso se acumulam a partir de suas interações com o jogo.

Qian et al (2016) examinou a literatura mais recente em relação à aprendizagem baseada em jogos e identificou 29 estudos que objetivaram as habilidades do século XXI como resultados. Seus resultados sugerem que uma abordagem de aprendizado baseada em jogos pode ser eficaz para facilitar o desenvolvimento de habilidades dos alunos no século XXI. $\mathrm{O}$ artigo também fornece informações valiosas para pesquisadores, designers de jogos e educadores em questões relacionadas ao design e à implementação de jogos educativos em geral.

Em Sourmelis (2016) apresentou o estado atual da arte na pesquisa empírica dos MMORPGs (Massive Multiplayer Online Role-Playing Game) de 2010 a 2016, buscando determinar o nível de maturidade do corpo de pesquisa de MMORPGs e para identificar falta de conhecimento, com relação as habilidades do século XXI. Os resultados revelaram um forte conjunto de evidências sugerindo que os MMORPGs são espaços nos quais uma variedade de habilidades do século XXI pode ser promovida.

Esses exemplos ilustram a abrangência e potencial do uso dos jogos digitais exercitando habilidades e competências, motivando a prática das habilidades do século XXI.

\section{Desafios e oportunidades de pesquisa}

Os profissionais utilizam avaliação de habilidades cognitivas, geralmente utilizando-se testes padronizados, como provas e testes, em áreas específicas, como as mais importante na predição educacional, devido sua maior facilidade de medição. Porém, a avaliação das habilidades socioemocionais continua um desafio, pois os alunos ainda estão em fase de desenvolvimento de tais habilidades e por não se conseguir medir apenas com escolhas de alternativas ou um texto escrito. O que deve ser ressaltado é que 
a avaliação deve considerar os dois tipos de habilidades para medir o desempenho do aluno [Silva Junior 2017].

Os jogos fornecem mecanismos que implantam situações-problema incentivado o jogador a tomar decisões e elaborar propostas para resolver situações naquela realidade, além de, através do raciocínio hipotético, antecipar os resultados de suas ações e prever o melhor cenário final. Em uma partida jogo digital típico, à medida que os jogadores interagem com o ambiente, os valores das diferentes variáveis específicas do jogo mudam. Por exemplo, se machucar em uma batalha reduz a saúde do personagem e encontrar um tesouro ou outro objeto aumenta seu estoque de mercadorias. Além disso, resolver grandes problemas nos jogos permite que os jogadores ganhem nível ou "suba de nível".

Agora considere o monitoramento de variáveis relevantes para a educação em diferentes níveis de granularidade nos jogos. Além de verificar o estado de saúde, os jogadores podem verificar seus níveis atuais de raciocínio, criatividade e trabalho em equipe, onde cada uma dessas competências é subdividida em elementos constituintes de conhecimento e habilidade (por exemplo, trabalho em equipe pode ser dividido em cooperação, negociação, e em habilidades de fluência). Se os valores estimados dessas competências forem muito baixos, o jogador provavelmente se sentiria obrigado a tomar medidas para impulsioná-los. Um dos principais desafios para os educadores que desejam empregar ou projetar jogos para apoiar o aprendizado e realizar inferências válidas - sobre o que o aluno sabe, acredita e pode fazer - a qualquer momento, em vários níveis e sem interromper o fluxo do jogo para não influenciar no engajamento e aprendizado na resolução dessas situações problema.

As estratégias de aprendizagem, seqüências de procedimentos ou atividades que se escolhem com o propósito de facilitar a aquisição, o armazenamento e/ou a utilização da informação [Khenissi et al 2016], podem ser ensinadas e aplicadas para alunos com baixo rendimento escolar, produzindo tanto uma melhoria imediata no uso das estratégias envolvidas no ensino como no rendimento escolar. As competências socioemocionais como: autoconceito, o sentimento de auto-eficácia, a ansiedade, a motivação e as atribuições de causalidade, entre outras, se relacionam ao uso apropriado das estratégias de aprendizagem [ Kang et al 2017 ].

Hwang et al. 2014 afirmou que pesquisadores identificaram os problemas e dificuldades da aplicação no uso de jogos, considerando que tal abordagem é um processo complexo no qual estratégias de ensino, aprendizagem e avaliações devem ser levados em consideração. É importante e necessário incluir estratégias de aprendizagem ou apoio para orientar os alunos, promovendo o próprio processo de aprendizagem, a regulação do raciocínio e a manutenção de um estado interno afetivo e motivacional que facilite a aprendizagem.

Ao projetar jogos de computador para apoiar os alunos em sala de aula, além de conteúdo educacional, eles também devem dar suporte aos benefícios pedagógicos, como estilo de aprendizagem, considerando também os com gêneros de jogo para o desenvolvimento de uma experiência de aprendizagem de qualidade em sala de aula. Khenissi et al (2016) realizou um mapeamento sobre a relação entre gêneros de jogos, técnicas de aprendizagem e estilos de aprendizagem em jogos educacionais de computador. No entanto, seu estudo propôs apenas o modelo potencial usado como uma 
possível diretriz conceitual para um estudo mais aprofundado, a fim de criar jogos de computador educativos eficazes. Um estudo mais aprofundado pode descobrir o ganho de benefício ao determinar os gêneros apropriados de jogo com estilos de aprendizagem.

Segundo seu gênero, os jogos podem ser aplicados para avaliar diferentes níveis de aprendizagem e habilidades [Khenissi et al 2016]. Os gêneros recorrentes para essa finalidade, devido suas características, são: (a) Adventure; (b) RPG; (c) Estratégia; (d) Simulação; (e) Esportes; (f) Casuais e; (g) Puzzle. Cada um desses gêneros trabalham sua narrativa e desenvolvimento de maneira diferente, por exemplo, jogos Adventure faz com que o jogador assuma o papel do protagonista em uma história interativa com exploração e resolução de quebra-cabeças, já os de estratégia exploram habilidades de pensamento e planejamento para alcançar a vitória.

Qian et al (2016) afirma que pouco se sabe sobre como os jogos podem influenciar a aquisição de habilidades do século XXI pelos alunos. Essas habilidades devem ser mapeadas de forma que consigamos ter uma definição exata, para que seus conceitos possam ser expressos nos jogos e assim, conseguir identificá-los na avaliação. Em alguns trabalhos é possível ver como identificar tais habilidades como: resolução de problemas [Shute et al. 2016], persistência e colaboração [ Shute e Ventura 2013]. Porém, algumas habilidades como criatividade [Kao et al. 2017], persistência [Shute et al. 2015], interpretação de conceitos [Shute et al. 2016 ], os autores realizaram testes externos para avaliar, devido a difícil modelagem de tais habilidades em seus trabalhos, As demais, não foram encontradas nos estudos ou ainda permanecem em trabalhos futuros.

Sourmelis (2016) informa que a maioria das pesquisas de MMORPGs se concentra na investigação da habilidade de comunicação (22\% das habilidades examinadas), enquanto criatividade e inovação, assim como a resolução de problemas e a alfabetização informacional, são amplamente inexploradas neste contexto.

Um ponto importante é saber como identificar no jogo, ações e eventos que trabalham uma habilidade específica, ou seja, definir padrões de ações, comportamentos dentro de certos ponto no jogo, que me dão informações suficientes para inferir valores sobre tal habilidade. Tal aspecto se torna relevante tando se for um jogo que irá ser desenvolvido ou se é um jogo previamente construído.

Com a captura dos eventos, escolhas e comportamentos fornecidos pelos jogador, pode-se utilizar conceitos de mineração de dados à procura de padrões consistentes, como regras de associação ou sequências temporais, para detectar relacionamentos entre as variáveis, detectando assim, novos agrupamentos de dados [Cheng et al. 2015]. Em específico, quando não se tem definidos padrões, costuma se usar métodos de mineração não supervisionado para realizar tal detecção. nesse sentido, vesse uma carência de trabalhos sobre o tema proposto.

Há também uma necessidade de saber se os padrões capturados e as classificações realizadas com o objetivo de medir as habilidades dos jogadores no mundo virtual, de fato, condizem com as habilidades no mundo real, necessitando de um cruzamento para verificar tal questionamento. De acordo com Khenissi et al (2016) alunos diferentes têm características diferentes, como estilos de aprendizagem, 
personalidade e traços cognitivos. Por isso, eles recebem e processam informações de maneira diferente. Recomendar jogos baseados em computador para aprendizagem, com base no gênero que melhor se adequa às características do aluno, pode aumentar a motivação dos alunos e levar a uma melhor experiência de aprendizado.

\section{Considerações finais}

As habilidades do século XXI incluem habilidades que foram identificadas como necessárias para o sucesso na sociedade e nos locais de trabalho do século XXI por educadores, líderes empresariais, acadêmicos e agências governamentais. Muitas dessas habilidades também estão associadas a um aprendizado mais profundo, baseado em habilidades como raciocínio analítico, resolução de problemas complexos e trabalho em equipe. Essas habilidades diferem das habilidades acadêmicas tradicionais, na medida em que não são primariamente baseadas em conhecimento.

Há uma necessidade associada de projetar e desenvolver avaliações válidas e confiáveis dessas novas habilidades. É necessário repensar a forma de como é realizado o ensino, visando propor diferentes formas de avaliação, diversificar os recursos utilizados, levando em consideração a cultura e os meios de expressão que permeiam os indivíduos presentes nesse processo, dessa forma, se oferece novas alternativas para os indivíduos interagirem e se expressarem para avaliar tais habilidades.

Nesse contexto, a introdução de atividades lúdicas como jogos digitais, com finalidades pedagógicas, é uma metodologia para ensinar de forma motivadora, possibilitando uma prática significativa daquilo que está sendo aprendido e potencializando a construção e avaliação do conhecimento. Porém, existem alguns desafios a serem preenchidos, um dos principais desafios para os educadores é usar a potencialidade dos jogos para apoiar o aprendizado e realizar avaliações, porém existe alguns desafios nesse cenário a criação e uso dos jogos devem utilizar estratégias de aprendizagem para orientar tanto os professores quanto os alunos aliado o gênero do jogo; modelagem de habilidades trabalhadas nesses jogos; Identificar que ações ou sequências de ações fornecidas pelo usuário reflete determinadas habilidades.

\section{Referências Bibliográficas}

Instituto Nacional de Estudos e Pesquisas Educacionais (1999). Exame Nacional do Ensino Médio: Documento Básico 2000. Brasília: INEP.

Cotta, R. M. M., Costa, G. D e Mendonça, E. T. (2015). Portfólios críticos-reflexivos: uma proposta pedagógica centrada nas competências cognitivas e metacognitivas. Interface (Botucatu). 19(54):573-88.

DeRosier, M. E., \& Thomas, J. M. (2017). Establishing the criterion validity of Zoo U's game-based social emotional skills assessment for school-based outcomes. Journal of Applied Developmental Psychology.

Gatti, B. (2005). Habilidades cognitivas y competencias sociales. Enunciación, 10(1):123-132. 
Kang, J., Liu, M., \& Qu, W. (2017). Using gameplay data to examine learning behavior patterns in a serious game. Computers in Human Behavior, 72, 757-770.

Kao, G. Y. M., Chiang, C. H., \& Sun, C. T. (2017). Customizing scaffolds for game-based learning in physics: Impacts on knowledge acquisition and game design creativity. Computers \& Education.

Khenissi, M. A., Essalmi, F., Jemni, M., Graf, S., \& Chen, N. S. (2016). Relationship between learning styles and genres of games. Computers \& Education, 101, 1-14.

Hwang, G. J., Hung, C. M., \& Chen, N. S. (2014). Improving learning achievements, motivations and problem-solving skills through a peer assessment-based game development approach. Educational Technology Research and Development, 62(2), 129-145.

OCDE, O. p. C. e. D. E. (2015). Skills for social progress: The power of social and emotional skills. Disponível em <https://goo.gl/YnywhV>. Acesso em: 22 mai. 2017.

Prensky, M. (2012). Aprendizagem baseada em jogos Digitais. SENAC SP.

Qian, M., \& Clark, K. R. (2016). Game-based Learning and 21st century skills: A review of recent research. Computers in Human Behavior, 63, 50-58.

Schrier, Karen. Using augmented reality games to teach 21 st century skills. In: ACM SIGGRAPH 2006 Educators program. ACM, 2006. p. 15.

Sena, S., Schmiegelow, S. S., Prado, G. M. B. C., Sousa, R. P. L. e Fialho, F. A. P. (2016). Aprendizagem baseada em jogos digitais: a contribuição dos jogos epistêmicos na geração de novos conhecimentos. Revista Novas Tecnologias na Educação. V. 14, No 1.

Serrano-Laguna, Á., Martínez-Ortiz, I., Haag, J., Regan, D., Johnson, A., \& Fernández-Manjón, B. (2017). Applying standards to systematize learning analytics in serious games. Computer Standards \& Interfaces, 50, 116-123.

Shute, V. and Ventura, M. (2013). Stealth assessment: Measuring and supporting learning in video games. MIT Press.

Shute, V. J., D'Mello, S., Baker, R., Cho, K., Bosch, N., Ocumpaugh, J., ... \& Almeda, V. (2015). Modeling how incoming knowledge, persistence, affective states, and in-game progress influence student learning from an educational game. Computers \& Education, 86, 224-235.

Shute, V. J., Wang, L., Greiff, S., Zhao, W., \& Moore, G. (2016). Measuring problem solving skills via stealth assessment in an engaging video game. Computers in Human Behavior, 63, 106-117.

Rosa, G. D. A. e Galvão, A. C. T. (2015). Conhecimento prévio e aprendizagem no ensino: implicações à luz do efeito reverso da expertise e de construtos computacionais da cognição. Ciências \& Cognição; Vol 20(2) 229-237.

Shute, V. and Ventura, M. (2013). Stealth assessment: Measuring and supporting learning in video games. MIT Press. 
Sourmelis, T., Ioannou, A., \& Zaphiris, P. (2017). Massively Multiplayer Online Role Playing Games (MMORPGs) and the 21st century skills: A comprehensive research review from 2010 to 2016. Computers in Human Behavior, 67, 41-48. 